\title{
OPTIMAL SWITCHING AMONG SEVERAL BROWNIAN MOTIONS
}

\author{
ROBERT J. VANDERBEI
}

\begin{abstract}
For $i=1, \ldots, d$, let $B_{s_{i}}^{i}$ be a one-dimensional Brownian motion on the interval $\left[0, a_{i}\right]$ with absorption at the endpoints. At each instant in time, we must decide to run some subset of these $d$ Brownian motions while holding the others fixed at their current state. The resulting process evolves in the rectangle $D=\left[0, a_{1}\right] \times \cdots \times\left[0, a_{d}\right]$. If, at some instant, we decide to freeze all of the Brownian motions, then a reward is received in accordance with this final position. We consider two types of reward functions.

First, we assume that the reward is zero everywhere in $D$ except along the $d$ edges that correspond to the coordinate axes. Along these edges it is given by $C^{3}$ strictly-concave functions $\gamma_{i}\left(x_{i}\right)$ which are zero at the endpoints 0 and $a_{i}$ of their domains. The optimal control for this problem has a simple description. Let

$$
\Gamma_{i}\left(x_{i}\right)=-\int_{0}^{x_{i}} u \gamma_{i}^{\prime \prime}(u) d u
$$

and put

$$
M_{i}=\left\{x \in D: \Gamma_{i}\left(x_{i}\right)=\max _{j} \Gamma_{j}\left(x_{j}\right)\right\} .
$$

We prove that the optimal control is: on $M_{i}$ run any Brownian motion except the $i^{\text {th }}$ one and stop at the first time an edge is reached.

The second class of reward functions are assumed to be zero everywhere except on the facets of $D$ that meet at the origin. On the $i^{\text {th }}$ such facet (i.e., where $\left.x_{i}=0\right)$, the reward function is the product of $\gamma_{j}\left(x_{j}\right)$ for $j \neq i$. Put

$$
N_{i}=\left\{x \in D: \Gamma_{i}\left(x_{i}\right)=\min _{j} \Gamma_{j}\left(x_{j}\right)\right\} .
$$
\end{abstract}

The optimal control is: on $N_{i}$ run the $i^{\text {th }}$ Brownian motion and stop when a facet of $D$ is reached.

Keywords. Optimal control, Hamilton-Jacobi-Bellman equation, local time, Brownian motion, smooth fit

AMS subject classifications. Primary 60G40, Secondary 60J45, 31C10

Abbreviated title. Optimal Switching Among Brownian Motions

\section{INTRODUCTION.}

For $i=1, \ldots, d$, let $B^{i}=\left\{B_{s_{i}}^{i}, s_{i} \geq 0\right\}$ be a one-dimensional Brownian motion on the interval $\left[0, a_{i}\right]$ with absorption at the endpoints. We assume that $B^{i}$ is adapted to a filtration $\mathcal{F}^{i}=\left\{\mathcal{F}_{s_{i}}^{i}, s_{i} \geq 0\right\}$ on the space $\Omega^{i}$ of continuous functions. Let $P_{x_{i}}^{i}, x_{i} \in\left[0, a_{i}\right]$, denote the probability measure associated with $B^{i}$ starting at the point $x_{i}$ and let $\mathbf{E}_{x_{i}}^{i}$ denote the corresponding expectation operator. We assume that the filtration $\mathcal{F}^{i}$ is complete with respect to every measure $P_{x_{i}}^{i}, x_{i} \in\left[0, a_{i}\right]$. 
The problem we study involves switching between these Brownian motions. We take as our sample space, the product $\Omega=\Omega^{1} \times \cdots \times \Omega^{d}$ and let the Brownian motions be independent by putting $P_{x}=P_{x_{1}}^{1} \times \cdots \times P_{x_{d}}^{d}\left(\mathbf{E}_{x}\right.$ will denote the corresponding expectation operator). A switching strategy $T$ is a family of random $d$-tuples,

$$
T=\left\{T(t)=\left(T_{1}(t), \ldots, T_{d}(t)\right), t \geq 0\right\}
$$

satisfying

$$
\begin{gathered}
T(0)=(0, \ldots, 0) \\
T_{i}(t) \text { is increasing in } t \text { for each } i
\end{gathered}
$$

and

$$
\left\{T_{1}(t) \leq s_{1}, \ldots, T_{d}(t) \leq s_{d}\right\} \in \mathcal{F}_{s_{1}}^{1} \times \cdots \times \mathcal{F}_{s_{d}}^{d} .
$$

The random variable $T_{i}(t)$ represents the amount of time the $i^{\text {th }}$ Brownian motion has been used up to time $t$. The interpretation of (4) is that, at time $t$, the total allocation of time between the $d$ processes must equal $t$. Condition (5) says that the switching strategy must be non-anticipating. The switched process $X^{T}$ is defined as

$$
X^{T}(t)=B_{T(t)}=\left(B_{T_{1}(t)}^{1}, \ldots, B_{T_{d}(t)}^{d}\right) .
$$

There are several possible criteria which may be optimized. Perhaps the most common is the accumulated discounted reward. In this case, we assume that each Brownian motion has a running reward function $r_{i}\left(x_{i}\right)$ and the problem then is to find the strategy $T^{*}$ which attains the following supremum:

$$
v(x)=\sup _{T} \mathbf{E}_{x} \int_{0}^{\infty} e^{-\lambda t} r\left(X^{T}(t)\right) \cdot d T(t),
$$

where $\lambda$ is a fixed positive constant, $r(x)=\left(r_{1}\left(x_{1}\right), \ldots, r_{d}\left(x_{d}\right)\right)$ and $r\left(X^{T}(t)\right) \cdot d T(t)$ represents the inner product between the vectors $r\left(X^{T}(t)\right)$ and $d T(t)$. This problem was studied by Karatzas [2], Mandelbaum [3] and Dalang [1] as a continuous time generalization of Gittins' index theorem for Markov chains (see, e.g., [6] Chapter 
14). Assuming each of the $r_{i}\left(x_{i}\right)$ are strictly increasing functions, they showed that there exist functions $\Gamma_{i}\left(x_{i}\right)$ which determine the optimal strategy as follows:

$$
T_{i}^{*}(t) \text { increases only when } X^{T^{*}}(t) \in M_{i}
$$

where

$$
M_{i}=\left\{x \in D: \Gamma_{i}\left(x_{i}\right)=\max _{j} \Gamma_{j}\left(x_{j}\right)\right\} .
$$

This strategy is called a follow the leader strategy since it runs process $i$ when $\Gamma_{i}\left(x_{i}\right)$ is the largest of all the functions $\Gamma_{j}\left(x_{j}\right)$. The functions $\Gamma_{j}\left(x_{j}\right)$ are called index functions.

A different optimization criterion was considered in [4]. For $d=2$, we studied the problem of finding $T^{*}(t)$ which attains the following supremum:

$$
v(x)=\sup _{T} \mathbf{E}_{x} f\left(X^{T}(\tau)\right),
$$

where $\tau$ is the first time the switched process $X^{T}$ exits a rectangle $D=\left[0, a_{1}\right] \times\left[0, a_{2}\right]$ and $f$ is a continuous pay-off function defined on the edges of $D$ and strongly concave (i.e. twice continuously differentiable and strictly concave) or linear on each edge. In the case where $f$ is zero except on the two edges that meet at the origin, it turns out that the optimal strategy has a simple description. Indeed, let $\gamma_{i}\left(x_{i}\right)$ denote the restriction of $f$ to the $x_{i}$ coordinate axis and put

$$
\Gamma_{i}\left(x_{i}\right)=-\int_{0}^{x_{i}} u \gamma_{i}^{\prime \prime}(u) d u=\gamma_{i}\left(x_{i}\right)-x_{i} \gamma_{i}^{\prime}\left(x_{i}\right) .
$$

In terms of the sets $M_{i}$ defined in (9), the optimal strategy satisfies:

$$
T_{i}^{*}(t) \text { increases only when } X^{T^{*}}(t) \notin M_{i} \text {. }
$$

Hence, the optimal strategy can be described as one which follows the loser.

The aim of this paper is to investigate how the above result generalizes to the case where $d>2$. Two possibilities come to mind. First, we could put concave data on the one-dimensional faces (i.e. edges) of $D$ that meet at the origin. In this case, we let $\tau$ be the first hitting time of the set of edges of $D$.

Theorem 1. Let

$$
f(x)=\left\{\begin{array}{cl}
\gamma_{1}\left(x_{1}\right) & \text { if } x=\left(x_{1}, 0, \ldots, 0\right) \\
\vdots & \\
\gamma_{d}\left(x_{d}\right) & \text { if } x=\left(0, \ldots, 0, x_{d}\right) \\
0 & \text { otherwise }
\end{array}\right.
$$




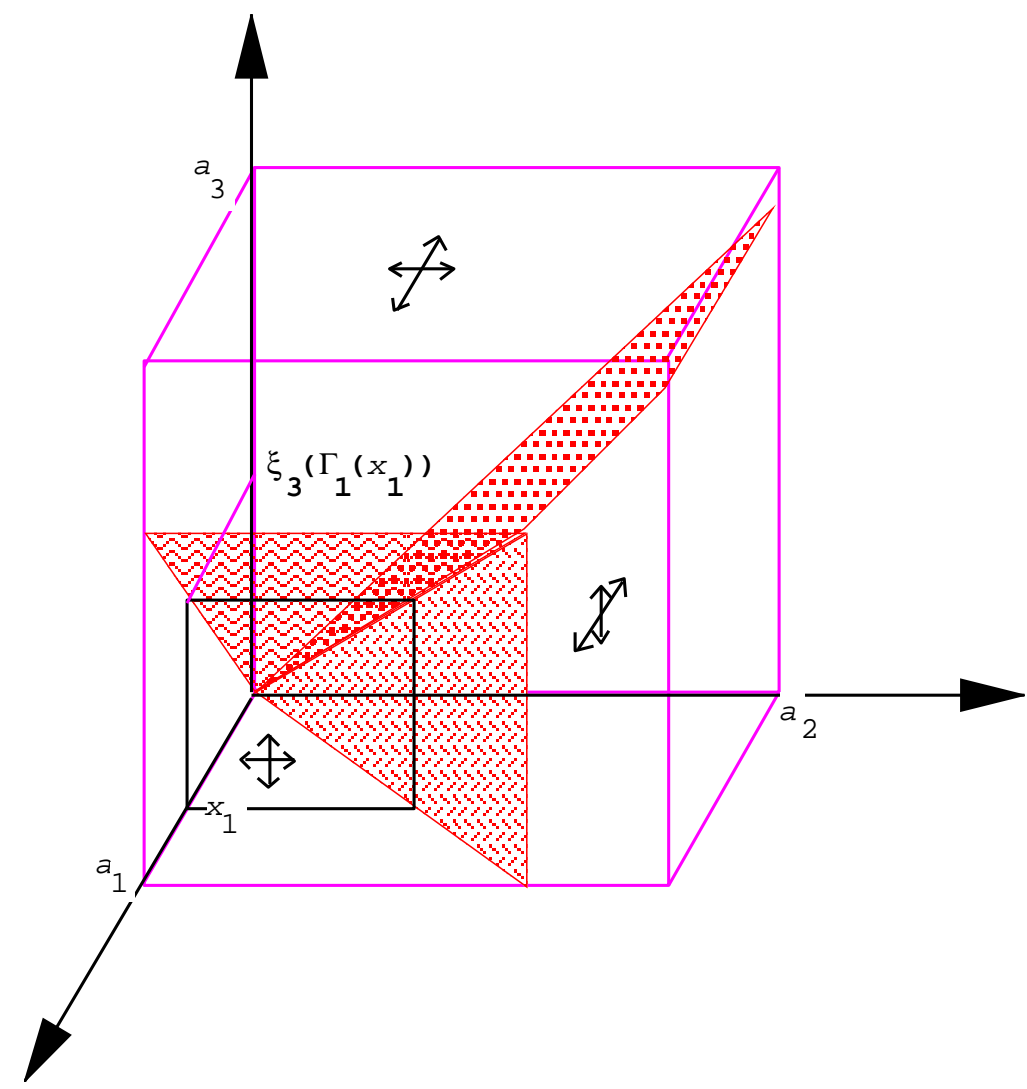

Figure 1. Following anybody but the leader. Here we show the switching surfaces in the special case where each are planar. The arrows indicate which Brownian motions can be run in each region.

where each $\gamma_{i}\left(x_{i}\right)$ is $C^{3}$, strictly-concave and vanishes at 0 and $a_{i}$. Optimal strategies exist. A strategy $T^{*}$ is optimal if and only if

$$
T_{i}^{*}(t) \text { increases only when } X^{T^{*}}(t) \notin M_{i}
$$

a.s. $P_{x}$, for all $x \in D$.

Hence, optimal strategies are ones which follow anybody but the leader. For $d=3$, the control regions are shown in Figure 1 (in the case where the borders between the switching regions are planar).

Alternatively, we could put (certain types of) concave data on the codimension one faces (i.e. facets) of $D$ that meet at the origin. In this case, $\tau$ is taken to be the first hitting time of a facet. 
Theorem 2. Let

$$
f(x)=\left\{\begin{array}{cl}
\gamma_{2}\left(x_{2}\right) \cdots \gamma_{d}\left(x_{d}\right) & \text { if } x=\left(0, x_{2}, \ldots, x_{d}\right) \\
\vdots & \\
\gamma_{1}\left(x_{1}\right) \cdots \gamma_{i-1}\left(x_{i-1}\right) \gamma_{i+1}\left(x_{i+1}\right) \cdots \gamma_{d}\left(x_{d}\right) & \text { if } x=\left(x_{1}, \ldots, x_{i-1}, 0, x_{i+1}, \ldots, x_{d}\right) \\
\vdots & \\
\gamma_{1}\left(x_{1}\right) \cdots \gamma_{d-1}\left(x_{d-1}\right) & \text { if } x=\left(x_{1}, \ldots, x_{d-1}, 0\right) \\
0 & \text { otherwise }
\end{array}\right.
$$

where, for each $i, \gamma_{i}\left(x_{i}\right)$ is $C^{3}$, strictly-concave and vanishes at 0 and $a_{i}$. There exists a unique (up to almost sure equivalence) optimal strategy. It satisfies:

$$
T_{i}^{*}(t) \text { increases only when } X^{T^{*}}(t) \in N_{i},
$$

where

$$
N_{i}=\left\{x \in D: \Gamma_{i}\left(x_{i}\right)=\min _{j} \Gamma_{j}\left(x_{j}\right)\right\} .
$$

So, for facet-data, the optimal strategy follows the loser. For $d=3$, the control regions are shown in Figure 2.

Remark: In Theorems 1 and 2 we assumed that the boundary data is three times continuously differentiable. Two derivatives should suffice. However, we will employ a change of variables in Sections 3 and 4 which necessitates our assumption of the existence of three derivatives. We believe that it should be possible to prove the results without using this change of variables, but the computations are more involved.

\section{Probabilistic Preliminaries.}

For the proofs of Theorems 1 and 2, we use the general theory of multiparameter processes. In this section, we review basic definitions and standard results. Our typical multiparameter process will be a real-valued function of $\left(B_{s_{1}}^{1}, \ldots, B_{s_{d}}^{d}\right)$ and so will always be adapted to the multiparameter filtration $\mathcal{F}=\left\{\mathcal{F}_{s_{1}}^{1} \times \cdots \times \mathcal{F}_{s_{d}}^{d}: s_{1} \geq\right.$ $\left.0, \ldots, s_{d} \geq 0\right\}$.

A multiparameter process $\mathcal{M}_{s_{1}, \ldots, s_{d}}$ is a supermartingale if it is adapted to $\mathcal{F}$, is integrable and satisfies the supermartingale property: for every $x \in D$ and for all $s_{1} \leq t_{1}, \ldots, s_{d} \leq t_{d}$,

$$
\mathbf{E}_{x}\left\{\mathcal{M}_{t_{1}, \ldots, t_{d}} \mid \mathcal{F}_{s_{1}}^{1} \times \cdots \times \mathcal{F}_{s_{d}}^{d}\right\} \leq \mathcal{M}_{s_{1}, \ldots, s_{d}} .
$$




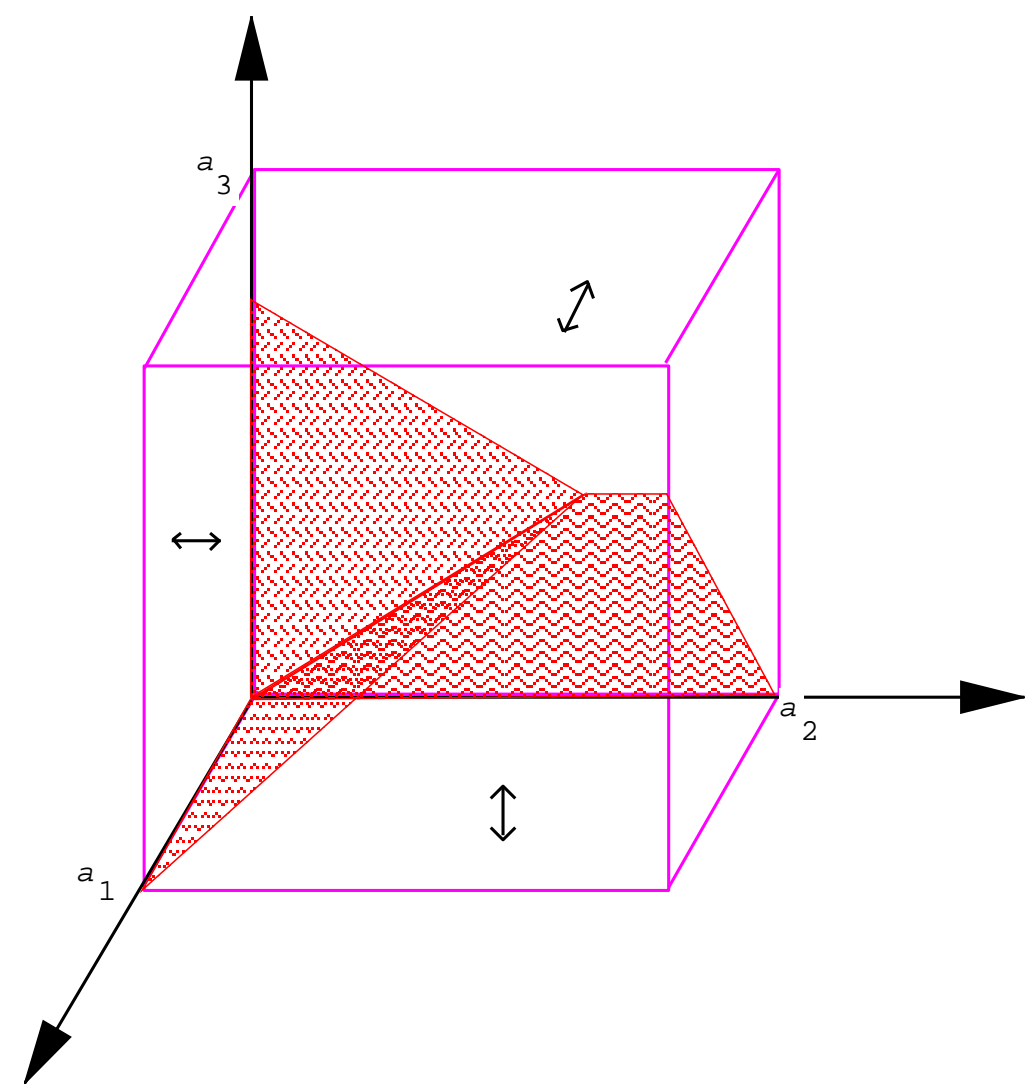

Figure 2. Following the loser. Here we show the switching surfaces in the special case where each are planar. The arrows indicate which Brownian motions can be run in each region.

It is a martingale if the above inequality is replaced by equality.

Associated with any switching strategy $T(t)$, there is a one-parameter filtration $\mathcal{F}^{T}=\left\{\mathcal{F}_{T(t)}: t \geq 0\right\}$ where $\mathcal{F}_{T(t)}$ is defined as the $\sigma$-algebra containing all measurable sets $C$ for which $C \cap\left\{T_{1}(t) \leq s_{1}, \ldots, T_{d}(t) \leq s_{d}\right\} \in \mathcal{F}_{s_{1}}^{1} \times \cdots \times \mathcal{F}_{s_{d}}^{d}$ for all $s_{1}, \ldots, s_{d}$. The switched process $X^{T}(t)$ is adapted to $\mathcal{F}^{T}$.

When we say that a multiparameter process is a martingale, we always mean that it is a martingale relative to $\mathcal{F}$. When we say that a one-parameter process, derived from a multiparameter process by following along a switching strategy $T(t)$, is a martingale, we mean that it is a martingale relative to $\mathcal{F}^{T}$.

A real-valued function defined on $D$ will be called multiconcave, if it is concave in each component separately. It is multilinear, if it is linear in each component separately. 
Proposition 3. (1) If $\mathcal{M}_{s}=\left(\mathcal{M}_{s_{1}}^{1}, \ldots, \mathcal{M}_{s_{d}}^{d}\right)$ is a multiparameter (super)martingale and $T(t)$ is a switching strategy, then $\mathcal{M}_{T(t)}$ is a (super)martingale.

(2) If $w$ is multilinear, then $w\left(X^{T}(t)\right)$ is a martingale for any strategy $T(t)$.

(3) If $w$ is multiconcave, then $w\left(X^{T}(t)\right)$ is a supermartingale for any strategy $T(t)$.

For $d=2$, these results follow from Propositions 2.4 and 3.1 in [5]. The proofs given in [5] apply to $d>2$ as well.

For the edge-data problem, let $E$ denote the set of edges of $D$ and for the facetdata problem, let $E$ denote the set of facets of $D$. Then, in either case, $\tau$ is the first hitting time of $E$.

Proposition 4. Let $w$ be a continuous, multiconcave function on $D$ that agrees with $f$ on $E$. If there exists a switching strategy $\tilde{T}(t)$ such that $w\left(B_{\tilde{T}(t \wedge \tau)}\right)$ is a martingale, then $w$ is the value function $v$ defined in $(10)$ and $\tilde{T}(t)$ is an optimal switching strategy.

Proof. Appealing to (3) of Proposition 3 and the optional sampling theorem, we conclude that

$$
w(x) \geq \mathbf{E}_{x} w\left(B_{T(\tau)}\right)=\mathbf{E}_{x} f\left(B_{T(\tau)}\right)
$$

for any switching strategy $T(t)$. Since $w\left(B_{\tilde{T}(t)}\right)$ is a martingale we see that

$$
w(x)=\mathbf{E}_{x} w\left(B_{\tilde{T}(\tau)}\right)=\mathbf{E}_{x} f\left(B_{\tilde{T}(\tau)}\right)
$$

From (15) and (16), we conclude that $w$ is the value function and that $\tilde{T}(t)$ is an optimal switching strategy.

Now, to prove Theorems 1 and 2, two tasks remain:

- Exhibit a function $w$ that is continuous, multiconcave and agrees with $f$ on $E$.

- Describe a switching strategy $\tilde{T}(t)$ for which $w\left(B_{\tilde{T}(t)}\right)$ is a martingale.

In order for $w\left(B_{\tilde{T}(t)}\right)$ to be a martingale it seems to be necessary that $w(x)$ be linear in at least one component at every point $x \in D \backslash E$. Hence, the function $w$ 
should be a solution to the following nonlinear Dirichlet problem:

$$
\begin{aligned}
\max _{i: 0<x_{i}<a_{i}} \frac{\partial^{2} w}{\partial x_{i}^{2}}(x)=0 & \text { for } x \in D \backslash E \\
w(x)=f(x) & \text { for } x \in E .
\end{aligned}
$$

In the next two sections, we construct twice continuously differentiable solutions to this differential equation.

Let $w$ denote the solution to (17), (18) corresponding to either the "edge-data" problem or the "face-data" problem. We finish this section by constructing a switching strategy $\tilde{T}(t)$ for which $w\left(B_{\tilde{T}(t)}\right)$ is a martingale. Consider any switching strategy $T(t)$. Since the functions $x_{i}$, and $x_{i} x_{j}$, for $j \neq i$, are multilinear, it follows from part 2 of Proposition 3 that

$$
X_{i}^{T}(t) \text { and } X_{i}^{T}(t) X_{j}^{T}(t)
$$

are martingales. Hence, for $t \geq 0$, the quadratic covariation between $X_{i}^{T}(t)$ and $X_{j}^{T}(t)$ vanishes:

$$
\left\langle X_{i}^{T}, X_{j}^{T}\right\rangle_{t}=0
$$

For each $i=1, \ldots, d$, the multiparameter process $\left(B_{s_{i}}^{i}\right)^{2}-s_{i}$ is a multiparameter martingale and so, by part 1 of Proposition 3, we see that

$$
\left(X_{i}^{T}(t)\right)^{2}-T_{i}(t)
$$

is a martingale. Hence, for $t \geq 0$, the quadratic variation of $X_{i}^{T}(t)$ is given by

$$
\left\langle X_{i}^{T}\right\rangle_{t}=T_{i}(t) .
$$

Since the function $w$ constructed in either of the next two sections is $C^{2}$, we can apply Ito's formula together with (19) and (20) to get that

$$
\begin{aligned}
w\left(X^{T}(t)\right)-w\left(X^{T}(0)\right)= & \sum_{i} \int_{0}^{t} \frac{\partial w}{\partial x_{i}}\left(X^{T}(s)\right) d X_{i}^{T}(s) \\
& +\sum_{i} \int_{0}^{t} \frac{\partial^{2} w}{\partial x_{i}^{2}}\left(X^{T}(s)\right) d T_{i}(s) .
\end{aligned}
$$

Theorem 12 in [3] establishes the existence of a strategy $\tilde{T}(t)$ that "follows the smallest index function":

$$
\tilde{T}_{i}(t) \text { increases only when } X^{\tilde{T}}(t) \in N_{i} \text {. }
$$

(In addition to existence, [3] also proves that the strategy is unique if the index processes $\Gamma_{i}\left(B_{s_{i}}^{i}\right)$ are simultaneously flat with probability zero. This condition is 
certainly met here for any pair of index processes and hence for any collection of them.) Whether considering the "edge-data" problem or the "facet-data" problem, in either case, the solution of (17), (18) constructed in the following sections has the property that

$$
\frac{\partial^{2} w}{\partial x_{i}^{2}}(x)=0 \text { for } x \in N_{i}
$$

(this follows from the fact that $N_{i} \subset M_{i}^{c}$ ). Combining (22) and (23), we see that the second sum in (21) vanishes and so $w\left(X^{\tilde{T}}(t)\right)$ is a martingale.

\section{Edge-Data.}

Let $w(x)$ denote a candidate for the value function $v(x)$ for the edge-data problem. Assuming that Theorem 1 correctly describes the optimal control regions, we see that $w(x)$ in the control region $M_{j}$ should be linear in every component except perhaps the $j^{\text {th }}$. That is, the restriction of $w$ to the intersection of the plane determined by a level set of $x_{j}$ and the control set $M_{j}$ should be multilinear. Hence, we can "sweep out" $w(x)$ to the boundary of $M_{j}$. For $d=2$ and for $x \in M_{1}$ this means that we can write:

$$
w\left(x_{1}, x_{2}\right)=\left(1-\frac{x_{2}}{\xi_{2}\left(\Gamma_{1}\left(x_{1}\right)\right)}\right) w\left(x_{1}, \xi_{2}\left(\Gamma_{1}\left(x_{1}\right)\right)\right)+\frac{x_{2}}{\xi_{2}\left(\Gamma_{1}\left(x_{1}\right)\right)} w\left(x_{1}, 0\right),
$$

where

$$
\xi_{i}(u)=\Gamma_{i}^{-1}\left(u \wedge \bar{u}_{i}\right)
$$

and

$$
\bar{u}_{i}=\Gamma_{i}\left(a_{i}\right) .
$$

Similarly, for $d=3$ and $x \in M_{1}$, the formula becomes

$$
\begin{aligned}
w\left(x_{1}, x_{2}, x_{3}\right)= & \left(1-\frac{x_{2}}{\xi_{2}\left(\Gamma_{1}\left(x_{1}\right)\right)}\right)\left(1-\frac{x_{3}}{\xi_{3}\left(\Gamma_{1}\left(x_{1}\right)\right)}\right) w\left(x_{1}, 0,0\right) \\
& +\frac{x_{2}}{\xi_{2}\left(\Gamma_{1}\left(x_{1}\right)\right)}\left(1-\frac{x_{3}}{\xi_{3}\left(\Gamma_{1}\left(x_{1}\right)\right)}\right) w\left(x_{1}, \xi_{2}\left(\Gamma_{1}\left(x_{1}\right)\right), 0\right) \\
& +\left(1-\frac{x_{2}}{\xi_{2}\left(\Gamma_{1}\left(x_{1}\right)\right)} \frac{x_{3}}{\xi_{3}\left(\Gamma_{1}\left(x_{1}\right)\right)} w\left(x_{1}, 0, \xi_{3}\left(\Gamma_{1}\left(x_{1}\right)\right)\right)\right. \\
& +\frac{x_{2}}{\xi_{2}\left(\Gamma_{1}\left(x_{1}\right)\right)} \frac{x_{3}}{\xi_{3}\left(\Gamma_{1}\left(x_{1}\right)\right)} w\left(x_{1}, \xi_{2}\left(\Gamma_{1}\left(x_{1}\right)\right), \xi_{3}\left(\Gamma_{1}\left(x_{1}\right)\right)\right) .
\end{aligned}
$$

For the general formula, the notation can be streamlined by observing that the $x_{1}$ in the argument list for $w$ can be written as $\xi_{1}\left(\Gamma_{1}\left(x_{1}\right)\right)$. In general, for $x \in M_{j}$, 
ROBERT J. VANDERBEI

$w\left(x_{1}, \ldots, x_{d}\right)$ can be written as a sum over those subsets $A$ of the set of indices $\{1,2, \ldots, d\}$ that contain $j$ :

$$
w\left(x_{1}, \ldots, x_{d}\right)=\sum_{A: j \in A} \prod_{i \notin A}\left(1-\frac{x_{i}}{\xi_{i}\left(\Gamma_{j}\left(x_{j}\right)\right)}\right) \prod_{i \in A, i \neq j} \frac{x_{i}}{\xi_{i}\left(\Gamma_{j}\left(x_{j}\right)\right)} w\left(T_{A} x_{j}\right),
$$

where $T_{A} x_{j}$ is the $d$-dimensional point whose coordinates are given by

$$
\left(T_{A} x_{j}\right)_{i}= \begin{cases}\xi_{i}\left(\Gamma_{j}\left(x_{j}\right)\right) & i \in A \\ 0 & i \notin A .\end{cases}
$$

Note that the product over $i \in A, i \neq j$ in (26) can actually be taken over all $i \in A$ since for $i=j$, the factor $x_{i} / \xi_{i}\left(\Gamma_{j}\left(x_{j}\right)\right)$ is just one.

Notations are greatly simplified if we change coordinates so that $x_{i}=\xi_{i}\left(u_{i}\right)$, for each $i$. Then the function $w$ becomes

$$
\tilde{w}\left(u_{1}, \ldots, u_{d}\right)=w\left(\xi_{1}\left(u_{1}\right), \ldots, \xi_{d}\left(u_{d}\right)\right),
$$

the domain $D$ becomes $\tilde{D}=\left\{\left(u_{1}, \ldots, u_{d}\right): u_{i} \leq \bar{u}_{i} \forall i\right\}$ and, for each $i$, the control region $M_{i}$ becomes

$$
\tilde{M}_{i}=\left\{\left(u_{1}, \ldots, u_{d}\right) \in \tilde{D}: u_{i}=\max _{j} u_{j}\right\} .
$$

Let $\tilde{w}_{j}$ denote the restriction of $\tilde{w}$ to $\tilde{M}_{j}$. Then,

$$
\tilde{w}_{j}\left(u_{1}, \ldots, u_{d}\right)=\sum_{A: j \in A} \prod_{i \notin A} q_{i}\left(u_{i}, u_{j}\right) \prod_{i \in A} \xi_{i}\left(u_{i}\right) \theta_{A}\left(u_{j}\right),
$$

where

$$
\begin{gathered}
q_{i}\left(u_{i}, u_{j}\right)=1-\frac{\xi_{i}\left(u_{i}\right)}{\xi_{i}\left(u_{j}\right)}, \\
\theta_{A}(u)=\frac{\tilde{w}\left(\tilde{T}_{A} u\right)}{\prod_{i \in A} \xi_{i}(u)},
\end{gathered}
$$

and

$$
\left(\tilde{T}_{A} u\right)_{i}= \begin{cases}\bar{u}_{i} \wedge u & i \in A \\ 0 & i \notin A .\end{cases}
$$

As long as we ensure that $\tilde{w}_{j}$ and $\tilde{w}_{k}$ patch together smoothly along their border $\tilde{M}_{j} \cap \tilde{M}_{k}$ then it follows that $\tilde{w}$ will be smooth throughout $\tilde{D}$. Since the change of variables (28) involves twice continuously differentiable functions (which follows from our assumption that the functions $\gamma_{i}$ are $C^{3}$ ), the smoothness of $\tilde{w}$ in $\tilde{D}$ translates back into the same smoothness of $w$ in $D$ (up to second order).

As we shall now show, stipulating first order smoothness across $\tilde{M}_{j} \cap \tilde{M}_{k}$ will force $\theta_{A}$ to be a specific function for each $A$. Stipulating second order smoothness will force the functions $\Gamma_{i}$ to be as defined in (11). 
Fix $j, k$ with $j \neq k$. First, we note that the values of $\tilde{w}_{j}$ and $\tilde{w}_{k}$ agree along $\tilde{M}_{j} \cap \tilde{M}_{k}$ :

$$
\left.\tilde{w}_{j}\right|_{u_{j}:=u_{k}:=u}=\sum_{A: j, k \in A} Q_{A} \Xi_{A} \theta_{A}(u)=\left.\tilde{w}_{k}\right|_{u_{j}:=u_{k}:=u},
$$

where $Q_{A}$ and $\Xi_{A}$ are abbreviations for the following expressions

$$
\begin{aligned}
Q_{A} & =\prod_{i \notin A} q_{i}\left(u_{i}, u\right) \\
\Xi_{A} & =\prod_{i \in A} \xi_{i}\left(u_{i}\right),
\end{aligned}
$$

and $\left.\tilde{w}_{j}\right|_{u_{j}:=u_{k}:=u}$ denotes the function $\tilde{w}_{j}\left(u_{1}, \ldots, u_{d}\right)$ evaluated at $u_{j}=u$ and $u_{k}=u$. It is easy to check that

$$
\begin{aligned}
\left.\frac{\partial \tilde{w}_{j}}{\partial u_{k}}\right|_{u_{j}:=u_{k}:=u}= & -\sum_{A: j \in A, k \notin A} \frac{\xi_{k}^{\prime}(u)}{\xi_{k}(u)} Q_{A \cup k} \Xi_{A} \theta_{A}(u) \\
& +\sum_{A: j, k \in A} \xi_{k}^{\prime}(u) Q_{A} \Xi_{A \backslash k} \theta_{A}(u)
\end{aligned}
$$

and

$$
\begin{aligned}
\left.\frac{\partial \tilde{w}_{k}}{\partial u_{k}}\right|_{u_{j}:=u_{k}:=u}= & \sum_{A: j \notin A, k \in A} \frac{\xi_{j}^{\prime}(u)}{\xi_{j}(u)} Q_{A \cup j} \Xi_{A} \theta_{A}(u) \\
& +\sum_{A: j, k \in A} \sum_{i \notin A} \frac{\xi_{i}\left(u_{i}\right) \xi_{i}^{\prime}(u)}{\xi_{i}^{2}(u)} Q_{A \cup i} \Xi_{A} \theta_{A}(u) \\
& +\sum_{A: j, k \in A} \xi_{k}^{\prime}(u) Q_{A} \Xi_{A \backslash k} \theta_{A}(u) \\
& +\sum_{A: j, k \in A} Q_{A} \Xi_{A} \theta_{A}^{\prime}(u) .
\end{aligned}
$$

Hence,

$$
\begin{aligned}
\left.\frac{1}{\prod_{i} \xi_{i}\left(u_{i}\right)}\left(\frac{\partial \tilde{w}_{k}}{\partial u_{k}}-\frac{\partial \tilde{w}_{j}}{\partial u_{k}}\right)\right|_{u_{j}:=u_{k}:=u}= & \sum_{A: j, k \in A} R_{A} \theta_{A}^{\prime}(u) \\
& +\sum_{A: j, k \in A} \sum_{i \notin A} \frac{\xi_{i}^{\prime}(u)}{\xi_{i}^{2}(u)} R_{A \cup i} \theta_{A}(u) \\
& +\sum_{A: j \notin A, k \in A} \frac{\xi_{j}^{\prime}(u)}{\xi_{j}^{2}(u)} R_{A \cup j} \theta_{A}(u) \\
& +\sum_{A: j \in A, k \notin A} \frac{\xi_{k}^{\prime}(u)}{\xi_{k}^{2}(u)} R_{A \cup k} \theta_{A}(u),
\end{aligned}
$$

where $R_{A}$ is an abbreviation for the following expression

$$
R_{A}=\frac{Q_{A}}{\prod_{i \notin A} \xi_{i}\left(u_{i}\right)}=\prod_{i \notin A}\left(\frac{1}{\xi_{i}\left(u_{i}\right)}-\frac{1}{\xi_{i}(u)}\right) .
$$


Combining the last three sums, we get

$$
\left.\frac{1}{\prod_{i} \xi_{i}\left(u_{i}\right)}\left(\frac{\partial \tilde{w}_{k}}{\partial u_{k}}-\frac{\partial \tilde{w}_{j}}{\partial u_{k}}\right)\right|_{u_{j}:=u_{k}:=u}=\sum_{A: j, k \in A} R_{A}\left(\theta_{A}^{\prime}(u)+\sum_{i \in A} \frac{\xi_{i}^{\prime}(u)}{\xi_{i}^{2}(u)} \theta_{A \backslash i}(u)\right)
$$

Hence, to guarantee that first derivatives of $\tilde{w}$ are continuous across $\tilde{M}_{j} \cap \tilde{M}_{k}$ for all $j \neq k$, it suffices to define $\theta_{A}$ so that

$$
\theta_{A}^{\prime}=-\sum_{i \in A} \frac{\xi_{i}^{\prime}}{\xi_{i}^{2}} \theta_{A \backslash i}
$$

for all $A$ containing two or more elements. If we let

$$
\bar{u}_{A}=\min _{j \in A} \bar{u}_{j}
$$

then $\tilde{T}_{A} \bar{u}_{A}$ lies on one of the "back faces" of $\tilde{D}$ (i.e. one of the components is at its upper bound) and so

$$
\theta_{A}\left(\bar{u}_{A}\right)=0 .
$$

Also, if $A$ contains exactly one element, say $j$, then we see from (28), (32), (33), and the fact that $w$ is to agree with $\gamma_{j}$ on the $j^{\text {th }}$ coordinate axis, that

$$
\theta_{j}(u)=\frac{\gamma_{j}\left(\xi_{j}(u)\right)}{\xi_{j}(u)}
$$

Hence, starting with sets $A$ of cardinality two and working up, each $\theta_{A}$ is uniquely determined by (36) and (37). Carrying out this recursion we get

$$
\theta_{A}(u)=\sum_{j \in A} \int_{\mathcal{R}_{A \backslash j}(u)}\left(\prod_{i \in A \backslash j} \frac{\xi_{i}^{\prime}\left(u_{i}\right)}{\xi_{i}^{2}\left(u_{i}\right)}\right) \theta_{j}\left(\max _{i \in A \backslash j} u_{i}\right) \prod_{i \in A \backslash j} d u_{i}
$$

where

$$
\mathcal{R}_{A}(u)=\left\{\left(u_{i}\right)_{i \in A}: u<u_{i} \leq \bar{u}_{i}\right\} .
$$


Finally, we need to check second derivatives. Carefully differentiating, we see that

$$
\begin{aligned}
\left.\frac{\xi_{k}^{2}(u)}{\xi_{k}^{\prime}(u)} \frac{1}{\prod_{i} \xi_{i}\left(u_{i}\right)} \frac{\partial^{2} \tilde{w}_{j}}{\partial u_{j} \partial u_{k}}\right|_{u_{j}:=u_{k}:=u}= & \sum_{A: j, k \in A} \sum_{i \notin A} \xi_{k}(u) \frac{\xi_{i}^{\prime}(u)}{\xi_{i}^{2}(u)} R_{A \cup i} \theta_{A}(u) \\
& +\sum_{A: j, k \in A} \xi_{k}(u) \frac{\xi_{j}^{\prime}(u)}{\xi_{j}(u)} R_{A} \theta_{A}(u) \\
& +\sum_{A: j, k \in A} \xi_{k}(u) R_{A} \theta_{A}^{\prime}(u) \\
& +\sum_{A: j \in A, k \notin A} \frac{\xi_{k}^{\prime}(u)}{\xi_{k}(u)} R_{A \cup k} \theta_{A}(u) \\
& -\sum_{A: j \in A, k \notin A} \sum_{i \notin A, i \neq k} \frac{\xi_{i}^{\prime}(u)}{\xi_{i}^{2}(u)} R_{A \cup i \cup k} \theta_{A}(u) \\
& -\sum_{A: j \in A, k \notin A} \frac{\xi_{j}^{\prime}(u)}{\xi_{j}(u)} R_{A \cup k} \theta_{A}(u) \\
& -\sum_{A: j \in A, k \notin A} R_{A \cup k} \theta_{A}^{\prime}(u) .
\end{aligned}
$$

Now substituting (36) into the above formula and reindexing so the $\theta$ 's always are subscripted with an $A$, we get

$$
\begin{aligned}
& \left.\frac{\xi_{j}^{2}(u)}{\xi_{j}^{\prime}(u)} \frac{\xi_{k}^{2}(u)}{\xi_{k}^{\prime}(u)} \frac{1}{\prod_{i} \xi_{i}\left(u_{i}\right)} \frac{\partial^{2} \tilde{w}_{j}}{\partial u_{j} \partial u_{k}}\right|_{u_{j}:=u_{k}:=u}=-\sum_{A: j \notin A, k \in A} \xi_{k}(u) R_{A \cup j} \theta_{A}(u) \\
& \text { - } \sum_{A: j \in A, k \notin A} \xi_{j}(u) R_{A \cup k} \theta_{A}(u) \\
& +\sum_{A: j, k \in A} \xi_{j}(u) \xi_{k}(u) R_{A} \theta_{A}(u) \\
& +\sum_{A: j, k \notin A,|A| \geq 1} R_{A \cup j \cup k} \theta_{A}(u) \\
& \text { - } R_{j \cup k} \theta_{j}^{\prime}(u) \frac{\xi_{j}^{2}(u)}{\xi_{j}^{\prime}(u)} .
\end{aligned}
$$

Interchanging the roles of $j$ and $k$, we can write down the analogous expression for $\tilde{w}_{k}$ and then subtract to get

$$
\left.\frac{\xi_{j}^{2}(u)}{\xi_{j}^{\prime}(u)} \frac{\xi_{k}^{2}(u)}{\xi_{k}^{\prime}(u)} \frac{1}{\prod_{i} \xi_{i}\left(u_{i}\right)}\left(\frac{\partial^{2} \tilde{w}_{j}}{\partial u_{j} \partial u_{k}}-\frac{\partial^{2} \tilde{w}_{k}}{\partial u_{j} \partial u_{k}}\right)\right|_{u_{j}:=u_{k}:=u}=R_{j \cup k}\left(\theta_{k}^{\prime}(u) \frac{\xi_{k}^{2}(u)}{\xi_{k}^{\prime}(u)}-\theta_{j}^{\prime}(u) \frac{\xi_{j}^{2}(u)}{\xi_{j}^{\prime}(u)}\right) .
$$

Now we are almost home. Recalling (38), we see that

$$
\theta_{k}(u)=\frac{\gamma_{k}\left(\xi_{k}(u)\right)}{\xi_{k}(u)}
$$


and so, using (11) and suppressing the dependent variable $u$, we get

$$
\theta_{k}^{\prime} \frac{\xi_{k}^{2}}{\xi_{k}^{\prime}}=\xi_{k} \gamma_{k}^{\prime}\left(\xi_{k}\right)-\gamma_{k}\left(\xi_{k}\right)=-\Gamma_{k}\left(\xi_{k}\right)=-u
$$

Hence, both sides of the difference on the right-hand side of (39) are equal to $-u$ and so the difference vanishes.

\section{Face-Data.}

Let $w(x)$ denote a candidate for the value function $v(x)$ for the face-data problem. As in the previous section, it is convenient to work in the system of coordinates defined by (28). Hence, the control region $N_{i}$ described in Theorem 2 becomes $\tilde{N}_{i}=\left\{\left(u_{1}, \ldots, u_{d}\right): u_{i}=\min _{j} u_{j}\right\}$.

Assuming that Theorem 2 correctly describes the optimal control regions, we see that $w(x)$ should be linear in $x_{i}$ on the set $N_{i}$. Hence, we can "sweep out" $w(x)$ to the boundary of $N_{i}$. Using the $u_{i}$ coordinates, this sweeping becomes

$$
\tilde{w}\left(u_{1}, \ldots, u_{d}\right)=\left(1-\frac{\xi_{i}\left(u_{i}\right)}{\xi_{i}\left(u_{j}\right)}\right) \prod_{k \neq i} \gamma_{k}\left(u_{k}\right)+\left.\frac{\xi_{i}\left(u_{i}\right)}{\xi_{i}\left(u_{j}\right)} \tilde{w}\left(u_{1}, \ldots, u_{d}\right)\right|_{u_{i}::=u_{j}},
$$

for

$$
\left(u_{1}, \ldots, u_{d}\right) \in \tilde{N}_{i, j}=\left\{\left(u_{1}, \ldots, u_{d}\right) \in \tilde{D}: u_{i} \leq u_{j} \leq \min _{k \neq i, j} u_{k}\right\} .
$$

First, we make sure that $\tilde{w}$ is twice continuously differentiable across the boundary

between $\tilde{N}_{i, j}$ and $\tilde{N}_{j, i}$. Let $\tilde{w}_{i, j}$ denote the restriction of $\tilde{w}$ to $\tilde{N}_{i, j}$ so that $\tilde{w}_{i, j}$ is given by the right-hand side in (40). From (40), we see that

$$
\left.\frac{\partial \tilde{w}_{i, j}}{\partial u_{i}}\right|_{u_{i}:=u_{j}:=u}=-\frac{\xi_{i}^{\prime}(u)}{\xi_{i}(u)} \gamma_{j}(u) \prod_{k \neq i, j} \tilde{\gamma}_{k}\left(u_{k}\right)+\left.\frac{\xi_{i}^{\prime}(u)}{\xi_{i}(u)} \tilde{w}_{i, j}\right|_{u_{i}:=u_{j}:=u}
$$

and

$$
\left.\frac{\partial \tilde{w}_{j, i}}{\partial u_{i}}\right|_{u_{i}:=u_{j}:=u}=\frac{\xi_{j}^{\prime}(u)}{\xi_{j}(u)} \gamma_{i}(u) \prod_{k \neq i, j} \tilde{\gamma}_{k}\left(u_{k}\right)-\left.\frac{\xi_{j}^{\prime}(u)}{\xi_{j}(u)} \tilde{w}_{j, i}\right|_{u_{i}:=u_{j}:=u}+\left.\frac{\partial}{\partial u} \tilde{w}_{j, i}\right|_{u_{i}:=u_{j}:=u},
$$

where $\tilde{\gamma}_{k}$ is defined by

$$
\left.\tilde{\gamma}_{k}\left(u_{k}\right)=\gamma_{k}\left(\xi_{k}\left(u_{k}\right)\right)\right) .
$$

Hence, for first derivatives to match we need

$$
\begin{aligned}
\left.\frac{\partial}{\partial u} \tilde{w}_{j, i}\right|_{u_{i}:=u_{j}:=u} & -\left.\left(\frac{\xi_{i}^{\prime}(u)}{\xi_{i}(u)}+\frac{\xi_{j}^{\prime}(u)}{\xi_{j}(u)}\right) \tilde{w}_{j, i}\right|_{u_{i}:=u_{j}:=u} \\
& =-\left(\frac{\xi_{i}^{\prime}(u)}{\xi_{i}(u)} \gamma_{j}(u)+\frac{\xi_{j}^{\prime}(u)}{\xi_{j}(u)} \gamma_{i}(u)\right) \prod_{k \neq i, j} \tilde{\gamma}_{k}\left(u_{k}\right) .
\end{aligned}
$$


At this point let us consider that part of the state space where $u_{1} \leq u_{2} \leq \cdots \leq$ $u_{d}$. From (40) and (41), we see that

$$
\tilde{w}\left(u_{1}, \ldots, u_{d}\right)=\left(1-\frac{\xi_{1}\left(u_{1}\right)}{\xi_{1}\left(u_{2}\right)}\right) G_{1}+\frac{\xi_{1}\left(u_{1}\right)}{\xi_{1}\left(u_{2}\right)} \theta_{2}\left(u_{2}\right)
$$

where $G_{i}$ is an abbreviation for

$$
G_{i}=\prod_{k>i} \tilde{\gamma}_{k}\left(u_{k}\right)
$$

and $\theta_{2}(u)=\tilde{w}\left(u, u, u_{3}, \ldots, u_{d}\right)$ is a solution of

$$
\theta_{2}^{\prime}-\left(\frac{\xi_{1}^{\prime}}{\xi_{1}}+\frac{\xi_{2}^{\prime}}{\xi_{2}}\right) \theta_{2}=-G_{2}\left(\frac{\xi_{1}^{\prime}}{\xi_{1}} \gamma_{2}+\frac{\xi_{2}^{\prime}}{\xi_{2}} \gamma_{1}\right) .
$$

Using the integrating factor $1 / \xi_{1} \xi_{2}$, we can solve for $\theta_{2}$ :

$$
\theta_{2}(u)=\xi_{1}(u) \xi_{2}(u)\left(\frac{\theta_{2}\left(u_{3}\right)}{\xi_{1}\left(u_{3}\right) \xi_{2}\left(u_{3}\right)}+G_{2} \int_{u}^{u_{3}}\left(\frac{\xi_{1}^{\prime} \tilde{\gamma}_{2}}{\xi_{1}^{2} \xi_{2}}+\frac{\xi_{2}^{\prime} \tilde{\gamma}_{1}}{\xi_{2}^{2} \xi_{1}}\right)\right),
$$

where we have suppressed the integration variable and its differential in the above integral. To keep notations in check, we will suppress integration variables and differentials several times in the following expressions. We hope this adds to the clarity of the formulas. Substituting this formula for $\theta_{2}$ into (42), we get

$$
\begin{aligned}
\tilde{w}\left(u_{1}, \ldots, u_{d}\right)= & \Xi_{1} G_{1} \int_{u_{1}}^{u_{2}} \frac{\xi_{1}^{\prime}}{\xi_{1}^{2}} \\
& +\Xi_{2} G_{2} \int_{u_{2}}^{u_{3}}\left(\frac{\xi_{1}^{\prime} \tilde{\gamma}_{2}}{\xi_{1}^{2} \xi_{2}}+\frac{\xi_{2}^{\prime} \tilde{\gamma}_{1}}{\xi_{2}^{2} \xi_{1}}\right) \\
& +\frac{\Xi_{2}}{\xi_{1}\left(u_{3}\right) \xi_{2}\left(u_{3}\right)} \theta_{3}\left(u_{3}\right),
\end{aligned}
$$

where $\Xi_{i}$ is an abbreviation for

$$
\begin{gathered}
\Xi_{i}=\prod_{k \leq i} \xi_{k}\left(u_{k}\right), \\
\theta_{3}(u)=\tilde{w}\left(u, u, u, u_{4}, \ldots, u_{d}\right),
\end{gathered}
$$

and the first term from (42) has been rewritten using the following simple identity

$$
\left(1-\frac{\xi_{1}\left(u_{1}\right)}{\xi_{1}\left(u_{2}\right)}\right)=\xi_{1}\left(u_{1}\right) \int_{u_{1}}^{u_{2}} \frac{\xi_{1}^{\prime}}{\xi_{1}^{2}} .
$$

Equation (41) can be used to get a directional derivative of $\tilde{w}$ at a point of the form $\left(u, u, u, u_{4}, \ldots, u_{d}\right)$. Writing down the analogous expressions obtained by considering the cases $u_{2} \geq \max \left(u_{1}, u_{3}\right)$ and $u_{1} \geq \max \left(u_{2}, u_{3}\right)$, we can get two more independent directional derivatives. From these three independent directional derivatives, it is not hard to see that $\theta_{3}$ satisfies the following differential equation

$$
\theta_{3}^{\prime}-\left(\frac{\xi_{1}^{\prime}}{\xi_{1}}+\frac{\xi_{2}^{\prime}}{\xi_{2}}+\frac{\xi_{3}^{\prime}}{\xi_{3}}\right) \theta_{3}=-\left(\frac{\xi_{1}^{\prime}}{\xi_{1}} \tilde{\gamma}_{2} \tilde{\gamma}_{3}+\frac{\xi_{2}^{\prime}}{\xi_{2}} \tilde{\gamma}_{1} \tilde{\gamma}_{3}+\frac{\xi_{3}^{\prime}}{\xi_{3}} \tilde{\gamma}_{1} \tilde{\gamma}_{2}\right)
$$


The integrating factor for this differential equation is $1 / \xi_{1} \xi_{2} \xi_{3}$. Integrating to solve for $\theta_{3}$ and substituting into (43), we get

$$
\begin{aligned}
\tilde{w}\left(u_{1}, \ldots, u_{d}\right)= & \Xi_{1} G_{1} \int_{u_{1}}^{u_{2}} \frac{\xi_{1}^{\prime}}{\xi_{1}^{2}} \\
& +\Xi_{2} G_{2} \int_{u_{2}}^{u_{3}}\left(\frac{\xi_{1}^{\prime} \tilde{\gamma}_{2}}{\xi_{1}^{2} \xi_{2}}+\frac{\xi_{2}^{\prime} \tilde{\gamma}_{1}}{\xi_{2}^{2} \xi_{1}}\right) \\
& +\Xi_{3} G_{3} \int_{u_{3}}^{u_{4}}\left(\frac{\xi_{1}^{\prime} \tilde{\gamma}_{2} \tilde{\gamma}_{3}}{\xi_{1}^{2} \xi_{2} \xi_{3}}+\frac{\xi_{2}^{\prime} \tilde{\gamma}_{1} \tilde{\gamma}_{3}}{\xi_{1} \xi_{2}^{2} \xi_{3}}+\frac{\xi_{3}^{\prime} \tilde{\gamma}_{1} \tilde{\gamma}_{2}}{\xi_{1} \xi_{2} \xi_{3}^{2}}\right) \\
& +\frac{\Xi_{3}}{\xi_{1}\left(u_{4}\right) \xi_{2}\left(u_{4}\right) \xi_{3}\left(u_{4}\right)} \theta_{4}\left(u_{4}\right),
\end{aligned}
$$

where

$$
\theta_{4}(u)=\tilde{w}\left(u, u, u, u, u_{5}, \ldots, u_{d}\right)
$$

Now it is easy to see how this process must continue. Ultimately, we get

$$
\tilde{w}\left(u_{1}, \ldots, u_{d}\right)=\sum_{i=1}^{d} \Xi_{i} G_{i} \int_{u_{i}}^{u_{i+1}} \sum_{k=1}^{i} \frac{\xi_{k}^{\prime}}{\xi_{k} \tilde{\gamma}_{k}} \prod_{j \leq i} \frac{\tilde{\gamma}_{j}}{\xi_{j}}
$$

where we have put $u_{d+1}=\bar{u}$ and used the fact that $\tilde{w}$ vanishes at $(\bar{u}, \ldots, \bar{u})$. On the other parts of the state space it is clear that we get an analogous formula with the indices changed so that an index $i$ is replaced with the index of the $i^{\text {th }}$ smallest $u$ value.

Now $\tilde{w}$ is defined everywhere in $\tilde{D}$ and is continuously differentiable throughout. It remains to show that it is also twice continuously differentiable. It is sufficient to show that second derivatives agree across the boundary between $\tilde{N}_{i, j}$ and $\tilde{N}_{j, i}$. Straightforward calculation shows that

$$
\left.\frac{\xi_{i} \xi_{j}}{\xi_{i}^{\prime} \xi_{j}^{\prime}}\left(\frac{\partial^{2} \tilde{w}_{i, j}}{\partial u_{i} \partial u_{j}}-\frac{\partial^{2} \tilde{w}_{j, i}}{\partial u_{i} \partial u_{j}}\right)\right|_{u_{i}:=u_{j}:=u}=\left(\frac{\xi_{i}(u)}{\xi_{i}^{\prime}(u)} \tilde{\gamma}_{i}^{\prime}(u)-\tilde{\gamma}_{i}(u)-\frac{\xi_{j}(u)}{\xi_{j}^{\prime}(u)} \tilde{\gamma}_{j}^{\prime}(u)+\tilde{\gamma}_{j}(u)\right) \prod_{k \neq i, j} \tilde{\gamma}_{k} .
$$

Since $\tilde{\gamma}_{i}(u)=\gamma_{i}\left(\xi_{i}(u)\right)$, we see that by $(11)$

$$
\frac{\xi_{i}(u)}{\xi_{i}^{\prime}(u)} \tilde{\gamma}_{i}^{\prime}(u)-\tilde{\gamma}_{i}(u)=\xi_{i}(u) \gamma_{i}^{\prime}\left(\xi_{i}(u)\right)-\gamma_{i}\left(\xi_{i}(u)\right)=-\Gamma_{i}\left(\xi_{i}(u)\right)=-u
$$

Similarly, the last two terms in parentheses in (44) add up to $u$ and so the right-hand side vanishes. This completes the proof that $w$ is twice continuously differentiable in $D$. 


\section{OTher EXAmples.}

Perhaps the most natural extension of the preceding results would be to consider more general biconcave data on the $d$ faces adjacent to the origin. It seems that such generalizations will be quite difficult. Indeed, for $d=3$ we considered the following data:

$$
f(x)= \begin{cases}\gamma_{0}\left(x_{2}\right) \gamma_{1}\left(x_{3}\right) & \text { if } x=\left(0, x_{2}, x_{3}\right) \\ \gamma_{0}\left(x_{3}\right) \gamma_{1}\left(x_{1}\right) & \text { if } x=\left(x_{1}, 0, x_{3}\right) \\ \gamma_{0}\left(x_{1}\right) \gamma_{1}\left(x_{2}\right) & \text { if } x=\left(x_{1}, x_{2}, 0\right) \\ 0 & \text { otherwise }\end{cases}
$$

where $\gamma_{0}$ and $\gamma_{1}$ are two different strongly concave functions which vanish at the endpoints of their domains. By considering a discretization of the Dirichlet problem (17), (18), we were able to apply the method of successive approximations to numerically solve for the value function and hence the optimal strategy. As in Figure 2, there are switching surfaces emanating from the three coordinate axes, but this time they do not meet along a single curve. In fact, the behavior of the optimal switching strategy is quite intricate inside a triangular tube enclosed by the three surfaces. It would be very interesting to understand more about the nature of examples such as this one.

\section{REFERENCES}

[1] R.C. Dalang. Randomization in the two-armed bandit problem. Ann. Prob., 18:218-225, 1990.

[2] I. Karatzas. Gittins indices in the dynamic allocation problem for diffusion processes. Ann. Prob., 12:173-192, 1984.

[3] A. Mandelbaum. Continuous multi-armed bandits and multi-parameter processes. Ann. Prob., 15:1527-1556, 1987.

[4] A. Mandelbaum, L.A. Shepp, and R.J. Vanderbei. Optimal switching between a pair of Brownian motions. Ann. Prob., 18:1010-1033, 1990.

[5] J.B. Walsh. Optional increasing paths. In Colloque ENST-CNET; Lecture Notes in Math 863, pages 172-201. Springer-Verlag, 1981.

[6] P. Whittle. Optimization over Time: Dynamic Programming and Stochastic Control. Wiley, 1982. 